\title{
Diagnóstico y monitoreo de leptospirosis en Latinoamérica
}

\section{Diagnosis and monitoring of leptospirosis in Latin America}

\section{Diagnóstico e monitorização de leptospirose em Latinoamérica}

\author{
Oscar Moreno Foglia ${ }^{1}$, Catalina Trujillo Salinas ${ }^{2}$, Cicero Antonio Maia Cavalcante ${ }^{3} \&$ \\ José Camilo Torres Romero ${ }^{4}$ \\ 'Licenciado en Biología, Médico Veterinario. ${ }^{2}$ Médico Veterinario. ${ }^{3}$ Licenciada em Ciências Biológicas \\ / Bacharelado em Ciencias Farmaêuticas. "Licenciado en Biología, Magister en Ciencias-Bioquímica, \\ Candidato a Doctor en Bioquímica y Biología Molecular UFC- Brasil.
}

1Dirección de Investigación, Fundación Muisca. Bogotá, Colombia. ²Programa de Medicina Veterinaria. Facultad de Medicina Veterinaria y de Zootecnia. Universidad Nacional de Colombia. Bogotá, Colombia. ${ }^{3}$ Licenciatura em Ciências Biológicas. Instituto Federal do Ceará (IFCE). Campus Jaguaribe - Ceará. Brasil. ${ }^{4}$ Programa de Ingeniería Ambiental.

Escuela de Ciencias Agrícolas, Pecuarias y del Medio Ambiente ECAPMA. Universidad Nacional Abierta y a Distancia UNAD. Bogotá. Colombia.

10gmorenof@unal.edu.co, ${ }^{2}$ catrujillos@unal.edu.co, ${ }^{3}$ ciceroamcavalcante@ifce.edu.br, 4jose.torres@unad.edu.co

\section{Resumen}

La leptospirosis es una enfermedad de gran importancia en el mundo debido a su componente zoonótico, el objetivo de esta investigación fue realizar una asociación entre la presencia de la enfermedad en animales domésticos, humanos y roedores silvestres en Latinoamérica. Se llevó a cabo una revisión bibliográfica haciendo énfasis en reportes asociados a roedores silvestres en los últimos 50 años. Como resultado se pudo establecer que los roedores silvestres seropositivos a la bacteria, en la mayoría de los casos, resultaban negativos en cultivo y los títulos eran bajos en comparación con la seroprevalencia en los animales domésticos, por lo que se deduce que son el principal factor de riesgo. Por tanto, es necesario establecer un control estricto en las explotaciones pecuarias, así como realizar estudios minuciosos, con el fin de disminuir los brotes en humanos e impedir la diseminación de la enfermedad a la fauna silvestre y la propagación de la enfermedad en humanos.

Palabras clave: Leptospira, roedores, zoonosis, diagnóstico, reservorio, Latinoamérica.

\section{Abstract}

Leptospirosis is a disease of great importance in the world because of its zoonotic component, the purpose of this research was to establish a relation between the presence of the disease among domestic, human and wild rodents in Latin America. A review of available bibliography was conducted with an emphasis on reports associated with wild rodents in the last 50 years. As a result it was established that wild rodents that were seropositive to the bacteria, in most cases, had negative culture results and titles were low compared to the seroprevalence in pets, it therefore follows that they are 
the main risk factor. In conclusion, it is necessary to establish a strict control on livestock farms as well as perform detailed studies, in order to reduce outbreaks in humans and prevent the spread of the disease to wildlife and humans.

Key-words: Leptospira, rodents, zoonoses, diagnosis, reservoir, Latin America.

\section{Resumo}

A leptospirose é uma doença de grande importância no mundo por causa de seu componente zoonótica, o objetivo desta pesquisa foi o de fazer uma associação entre a presença da doença em animais domésticos, humanos e roedores silvestres na América Latina. Foi realizada uma revisão da literatura com ênfase em relatórios associados a roedores silvestres nos últimos 50 anos. Como resultado pudo estabelecer-se que os roedores silvestres seropositivos à bacteria, na maioria dos casos, resultavam negativos em cultura e os títulos eram baixos em relação com a seroprevalência nos animais domésticos, pelo qual se deduze que são o principal fator de risco. Portanto, é necessário estabelecer um control rigoroso nas explotações pecuarias bem como realizar estudos detalhados a fim de disminuir a incidência em humanos e evitar a diseminação da doença à fauna Silvestre.

Palavras-chave: Leptospira, roedores, zoonoses, diagnóstico, reservatório, América Latina.

\section{Introducción}

Los miembros del genero Leptospira son bacterias helicoidales que pueden existir como saprofitos de vida libre o en asociación con un diverso rango de hospederos animales (Adler \& Faine, 2006; Cespedes, 2005). La leptospirosis es una epizoodemia de gran importancia en el mundo. Produce grandes pérdidas económicas a nivel pecuario y repercusiones graves para la salud humana. A pesar de esto son pocos los estudios que se realizan de los ciclos silvestres de esta enfermedad y cómo es su relación con el ecosistema. (Sandow \& Ramírez, 2005; Cespedes, 2005; Bharti et al., 2006).

En países tropicales como Colombia las condiciones ambientales y productivas favorecen la transmisión y replicación de la enfermedad; variables como la precipitación, humedad, temperatura, estructura y composición del suelo, así como la irrupción de la frontera agrícola a ecosistemas cerrados son los factores más favorables para su presentación (Morales, Guzmán \& Beltrán, 1978). La principal fuente de transmisión al humano es el contacto con orina y heces de roedores, caninos, porcinos y bovinos durante la enfermedad activa o en su fase de portadores asintomáticos. Es imprescindible tomar medidas efectivas para el control de ésta enfermedad, debido a su fácil transmisión entre especies. Identificar el serogrupo o serovar actuante es imprescindible, puesto que la presencia de un serovar o serogrupo determinado depende principalmente de la existencia de su hospedero definitivo y según sea el hospedero las medidas de control serán diferentes.

Según lo observado en otras investigaciones, los roedores hacen parte esencial del ciclo de transmisión de la Leptospira, por lo que se debe hacer especial énfasis en la identificación de éstos cómo factores de riesgo dentro del ecosistema; por esta razón en el presente artículo se pretende compilar información sobre la identificación de la bacteria en roedores silvestres especialmente en cuyes (Cavia spp.) en diferentes zonas de América Latina teniendo en cuenta su rol epidemiológico en los ciclos silvestres de la enfermedad (Petrakovsky et al., 2014).

\section{Planteamiento de la temática}

Se realizó la recopilación de los principales artículos publicados acerca de las bacterias del género Leptospira que producen enfermedades clínicas 
frecuentes en Latinoamérica. El artículo incluye los trabajos publicados en los últimos 50 años, por cualquier institución o investigador individual y en cualquier idioma, se presenta información sobre el organismo patógeno, hospederos roedores, epidemiología, diagnóstico del patógeno y factores de riesgo.

\section{El organismo}

El género Leptospira comprende espiroquetas delgadas de aproximadamente 6 a $20 \mu \mathrm{m}$ de longitud y 0,1 a 0,2 $\mu \mathrm{m}$ de diámetro. En este género se incluyen especies patogénicas y saprofitas que se encuentran agrupadas en dos especies: $L$. biflexa y $L$. interrogans que contienen a su vez más de 210 serovares y 23 serogrupos de acuerdo a sus antígenos determinantes (Faine, Adler, Bolin \& Perolat, 1999; Levett, 2001). Se describe el aspecto morfológico de estos microorganismos que presentan en su parte polar dos flagelos que se insertan en el espacio periplásmico y cuya principal función es la locomoción (Picardeau, Brenot \& Saint Girons, 2001; Barbosa et al., 2006). Tienen además una envoltura celular similar a las bacterias Gram negativas, que consiste en una membrana citoplasmática y otra externa. Sin embargo, la capa de péptidoglicano está asociada con la membrana citoplasmática en vez de la membrana externa, algo que es único de las espiroquetas. Son organismos aerobios obligatorios y se les considera bacterias hidrófilas ya que en la mayoría de estudios se le relaciona con ambientes de alta humedad relativa y $\mathrm{pH}$ neutral (Sacsaquispe, Gleny \& Cespedes, 2003).

\section{Patogénesis}

Leptospira es un patógeno que infecta gran diversidad de mamíferos incluyendo humanos y animales domésticos. En estas especies la enfermedad se caracteriza por ser de tipo sistémico y provoca fiebre, insuficiencia renal y hepática, manifestaciones respiratorias y en algunas especies problemas reproductivos (Ballard et al., 1986). Aunque en la mayoría de especies se presenta enfermedad, en algunos casos una vez recuperadas pueden portar el microorganismo de forma asintomática y excretarlo por vía renal durante largos periodos al medio ambiente favoreciendo su diseminación (Levett et al., 2005; Levett, Morey, Galloway \& Steigerwalt, 2006).

Los hospederos son susceptibles de adquirir la infección al entrar en contacto la piel lesionada o las mucosas con orina o tejido de animales infectados. Especies de roedores cómo el ratón doméstico (Mus musculus), rata novergica (Rattus novergicus) y rata común (Rattus rattus) se han relacionado como reservorios de ciertos serovares de Leptospira (Bharti et al., 2003; Sacsaquispe, Glenny \& Cespedes, 2003). El microorganismo entra directamente al torrente sanguíneo. Los serovares apatógenos fallan en la multiplicación y son rápidamente eliminados por el sistema inmune del huésped dentro del primer y segundo día. Los organismos virulentos se multiplican hasta que son opsonizados y fagocitados durante la etapa en que los anticuerpos aglutinantes son apenas detectables por prueba de microaglutinación; éstas son rápidamente eliminadas por el sistema retículoendotelial. La tasa de crecimiento in vivo es equivalente a seis $u$ ocho horas en infecciones experimentales. En animales de experimentación altamente susceptibles, el crecimiento continúa hasta que altas concentraciones de Leptospira son encontradas en todos los tejidos, especialmente sangre, hígado, riñón, pulmón, cerebro y glándulas adrenales. En este momento los signos clínicos y los cambios patológicos en muchos tejidos son evidentes. Si el animal está preñado, el feto o fetos pueden infectarse durante la fase de leptospiremia. La patogénesis de la infección y los cambios patológicos en el feto se asemejan esencialmente a los observados en animales adultos, pero son relativamente más severos debido a la falta de inmunidad en el feto (Faine et al., 1999).

En la mayoría de los casos la infección con Leptospira en los roedores es asintomática o genera signos leves de enfermedad (Percy \& Barthold, 2007), por lo que el cuadro clínico en estos animales es poco descrito. En humanos la infección ha sido caracterizada como bifásica. El periodo de 
incubación oscila entre 2 y 26 días con una media de 10 días. El primer estadio es la fase septicémica que tiene una duración aproximada de 4 a 7 días con presentación de fiebre, cefalea, dolores osteomusculares y depresión. Durante esta fase es posible el aislamiento de la bacteria en sangre, orina y otros fluidos (Chinchilla, Boza \& Sáens, 1996). La segunda fase corresponde a la fase inmune cuya duración es de 4 a 30 días, para este caso la bacteria ha abandonado el torrente sanguíneo y ha infectado otros órganos como riñones, hígado, humor acuoso, entre otros (Chinchilla, Boza \& Sáenz, 1996).

La patogenicidad de la Leptospira no está del todo conocida, sin embargo se han realizado muchos estudios relacionados con los factores de virulencia que pueden dar una idea de los mecanismos de daño en los hospederos. Igualmente se ha demostrado que la inmunidad del hospedero tiene un papel importante en la patogenicidad de la bacteria puesto que se ha observado que hay una gran variedad de manifestaciones clínicas (Bharti et al., 2003; Sacsaquispe, Glenny \& Cespedes, 2003). Los mecanismos de patogenicidad pueden dividirse en dos tipos: Los efectos directos de Leptospira y la respuesta inmune del hospedero. Uno de los mecanismos de virulencia es la motilidad de la bacteria y su capacidad para moverse en medios viscosos (Faine et al., 1999). Esta característica es importante durante la fase de infección y diseminación de la bacteria desde el sitio de entrada hasta los órganos blanco. La actividad hemolítica, fosfolipasa y esfingomielinasa se ha demostrado in vitro aunque la relevancia de éstos no ha sido bien establecida (Bharti et al., 2003).

Se ha demostrado la síntesis de lipopolisacárido (LPS) en las interacciones con células in vitro. También se ha encontrado la existencia de hemolisinas y la capacidad de adherencia de ciertas cepas y de diversos serotipos a los fibroblastos de ratón; esta propiedad es superior cuando se trata de cepas virulentas. En otros estudios in vitro se evidenció que las leptospiras tienen capacidad de penetración intracelular en células endoteliales humanas; aunque tal propiedad invasora no ha podido demostrarse in vivo, explicaría la vasculitis hemorrágica petequial observada en las formas graves de leptospirosis humana (Dooley et al., 1976; Cerqueira \& Picardeau, 2009).

\section{Epidemiología}

La leptospirosis es una enfermedad de distribución mundial. La incidencia en humanos es más alta en el trópico aunque en países estacionales también se presenta la enfermedad. Las tasas de incidencia están subestimadas ya que no hay un reporte de diagnóstico eficaz y en regiones endémicas es común que los casos tengan signos leves o sean asintomáticos (Bovet et al., 1999; Ashford, Kaiser \& Spiegel, 2000). La mayoría de los casos ocurren en adultos jóvenes, principalmente personas con riesgos ocupacionales que los hacen estar en contacto con aguas contaminadas con leptospiras (agricultores en terrenos inundados, veterinarios, mineros y trabajadores de alcantarillados, entre otros), así como personas con exposición recreacional (nadadores, acampadores y pescadores, entre otros). Se ha observado la presencia de brotes epidémicos en soldados que durante su entrenamiento han ingerido agua contaminada (Bovet et al., 1999). Las características socioeconómicas de las poblaciones tal y como lo describen Pereira \& Andrade (1990) en los habitantes de los barrios bajos de Río de Janeiro, favorece la proliferación del reservorio principal (Corwin et al., 1990).

Los roedores fueron los primeros reservorios conocidos de la bacteria relacionados con la transmisión de la enfermedad a los humanos (Matthias \& Levett, 2002). Desde 1963 se han hecho estudios de asociación entre Leptospira y los roedores suramericanos. Blood, Szifres \& Moya (1963) demostraron la infección por la $L$. pomona en Cavia pamparum en la provincia de Corrientes, Argentina, por medio de cultivo y microaglutinación, asociada a un brote en ganado bovino. Se colectaron 282 muestras de las cuales 25 dieron positivas por serología (9\%) y 11 para cultivo (4\%). Los autores exponen la alta correlación entre el mantenimiento del ciclo de la enfermedad en los 
bovinos y la infección en los cuyes ya que pese a que los muestreos se realizaron en intervalos de tiempo diferentes siempre hubo animales seropositivos en los potreros problema.

En los años 1966 y 1967 Liceras (1975), llevó a cabo un estudio de identificación de serovares de Leptospira en Perú. Además de las muestras en animales domésticos se tomaron muestras serológicas de seis cobayos silvestres (Cavia aperea festina) y siete roedores no identificados. De los 6 cobayos 4 resultaron seropositivos para los serovares pomona-autumnalis-pyrogenes, pomonaautumnalis-butembo, pomona-butembo-copenhageni, pomona-grippotyphosa-butembo; con títulos que variaron de 1:100 y 1:3000.

Cordeiro \& Sulzer (1981) en Brasil, llevaron a cabo un estudio en el que evaluaron la infección por Leptospira en diferentes especies de animales silvestres del sudeste del país; de 43 especies examinadas 6 especies de roedores fueron asociadas como portadoras de la bacteria: Mus musculus, Akodon arviculoides, Rattus rattus, Rattus norvegicus, Nectomys squamipes y Oryzomys nigripes. Del total de 742 animales analizados 88 fueron seropositivos a uno o varios serovares de la bacteria donde: de 173 Mus musculus resultaron positivos $63(36,41 \%)$ a los serovares ballum, pomona y tropica; de 307 Akodon arviculoides fueron positivos 14 $(4,56 \%)$ para el serovar pomona y 2 más no identificados. De 189 Rattus rattus, 5 (2,64\%) fueron positivas a serovar pomona y 1 no identificado; de 21 Rattus norvegicus, 2 (9,52\%) fueron positivas a un serovar no identificado. De 42 Nectomys squamipes, 2 (4,76\%) animales fueron positivos para el serovar australis y de 10 Oryzomys nigripes, 2 (20\%) fueron seropositivos al serovar pomona. Este estudio permitió identificar un total de 8 diferentes serovares y nuevas especies reservorio de la bacteria en Brasil y predecir factores de riesgo para los animales domésticos y los humanos.

Más tarde, en 1982 se investiga la presencia de la infección por Leptospira en 106 roedores silvestres capturados en el área rural de la provincia de Valdivia, Chile. En esta ocasión se empleó la tinción de Levaditi en el tejido renal para detectar la leptospira microscópicamente. También se realizó un estudio seroprospectivo en esos pequeños mamíferos, detectándose que 47 (16\%) eran positivos, predominando los anticuerpos frente a los serovares más frecuentemente constatados en los animales domésticos de la zona, tales como pomona, sejroe, copenhageni y tarassovi, entre otros, prevaleciendo la infección en Abrothrix Iongipilis, Abrothrix olivaceus y Oligoryzomys longicaudatus (Riedemann \& Zamora, 1982).

Durante estudios posteriores en Chile, se capturaron 784 roedores múridos silvestres en zonas urbanas y rurales de Valdivia durante 3 años, esto permitió establecer que la prevalencia cruda para esta zona fue de $37.8 \%$; también se pudo comprobar que éstos estaban infectados en un porcentaje superior en el área rural (41.4\%) que los cazados en la ciudad (24.2\%). De acuerdo al diagnóstico efectuado por serología, aislamiento y tinciones inmunoquímicas y según la aglutinación microscópica el serovar pomona fue el de mayor prevalencia seguido en orden descendente por canicola, hardjo, javanica, icterohaemorrhagiae y tarassovi. Según la especie resultaron positivos: 20/97 (20.6\%) ejemplares de $M$. musculus, $87 / 175$ (49,7\%) de A. longipilis, 91/206 (44.2\%) de A. olivaceus, 77/191 (40.3\%) de $O$. longicaudatus, $18 / 85$ (21.2\%) de $R$. rattus, $2 / 27(7.4 \%)$ de $R$. norvegicus , $1 / 2(50 \%)$ de $G$. valdivianus y $0 / 1(0.0 \%)$ de $A$. microtus. Se pudo apreciar que la prevalencia varió notablemente de acuerdo a la estación del año en que se capturó el roedor, siendo mayor en invierno con un $41.4 \%$, para disminuir en otoño y primavera a un $37.3 \%$ y ser la más baja en verano con el $29.8 \%$. Situaciones semejantes se han descrito en otros países, evidenciando que los valores de infección son bajos en los roedores adultos durante los meses de verano, pero se incrementan notablemente a comienzos de otoño hasta el inicio de la primavera (Zamora, Riedemann \& Cabezas, 1995; Zamora, 1998; Zamora \& Riedemann, 1999). 
En un estudio realizado por Bunnel (2002) en el Amazonas peruano, en donde se tomaron muestras de riñón de diferentes especies de animales silvestres, se encontró que de las 72 muestras de roedores evaluadas por PCR la seroprevalencia para Leptospira en estos animales era del $20 \%$. Vanasco, Sequeira, Sequeira \& Tarabla (2003) realizaron un estudio en la ciudad de Santa Fe, Argentina, en el que se asocia la presencia de la enfermedad junto a la seropositividad de los roedores y las condiciones ambientales. En el estudio se capturaron 214 roedores domésticos y silvestres de los cuales se procesaron 201 muestras. Los resultados de este estudio arrojan una prevalencia cruda por seropositividad (ELISA positivo) del $48 \%$. En las áreas de corredores naturales, los aislamientos correspondían al el serovar ballum, mientras que en la zona urbana se aisló el serotipo icterohemorrágica. Con respecto a las especies de roedores, la familia Murinae fue la más común; M. musculus, fue la especie más capturada, para ésta, de 92 muestras probadas para Elisa el 34\% resultó positivo. En el caso del cultivo de las 43 muestras el $44 \%$ se logró cultivar y correspondían al serogrupo ballum. $R$. novergicus mostró una prevalencia serológica del 48\% (23 muestras) y el diagnóstico por cultivo fue del $12 \%$ (17 muestras) aislándose el serogrupo ballum. $R$. rattus resultó positiva a serología en un 38\% (32 muestras) y el aislamiento por cultivo fue del 18\% (17 muestras), en las que se aisló el serotipo icterohemorrágica. Para la familia Sigmodontinae se capturaron individuos de las especies $A$. azarae, $C$. laucha, C. musculinus, $H$. brasiliesis, y $O$. flavescens en las que se encontraron seroprevalencias de 41\% (29 muestras), 0\% (2 muestras), 0\% (2 muestras), 100\% (2 muestras) y $84 \%$ (19 muestras), respectivamente; mientras que para el cultivo todas las muestras resultaron negativas. Los autores concluyen que no está del todo claro el papel de los roedores silvestres en el ciclo de la leptospirosis en Santa Fe, ya que aunque algunos fueron seropositivos en el cultivo, inmunohistoquímica y tinción diferencial de plata fueron negativos.
Gressler et al. (2009) llevaron a cabo un estudio serológico en 5 cuyes (Cavia aperea) capturados en el campus de la Universidad de Santa María, provincia de Santa María, estado de Rio Grande do Sul, Brazil. Los serovares de Leptospira interrogans estudiados fueron: bratislava, butembo, canicola, grippotyphosa, hardjo, icterohaemorrhagiae, pomona, y wolffi. Cómo resultados se obtuvo que 4 de los 5 cuyes evaluados presentaban infección mixta por los serovares de L. interrogans con títulos de 1:100. Dos roedores eran positivos serológicamente a hardjo y butembo y los otros a hardjo y bratislava. Sin embargo, los animales no presentaban signos clínicos de la enfermedad.

En 2011 Montes et al. realizaron un estudio en ardillas nuca blanca (Sciurus stramineus) cuyo objetivo fue detectar la frecuencia de anticuerpos contra Leptospira sp. en la población silvestre capturada en el zoológico Parque de Las Leyendas, Lima, Perú. Se colectaron 35 muestras de suero de ardillas de ambos sexos, de diferentes edades y zonas del zoológico y se evaluaron mediante la prueba de microaglutinación. El 82.3\% (29/35) de animales presentó anticuerpos contra Leptospira sp., donde el $60 \%$ correspondió al serovar icterohemorraghiae, seguidos por georgia (31.4\%), canicola (5.7\%), y australis (2.8\%). No hubo diferencia estadística entre la presencia de anticuerpos contra Leptospira sp. y las variables sexo, edad y área de captura de las ardillas.

Méndez et al. (2013) determinaron la distribución de anticuerpos antileptospira en roedores silvestres y en tres especies domésticas que comparten el mismo hábitat en un área endémica de leptospirosis bovina al NE de México. Se analizaron muestras de suero de 24 roedores, 220 bovinos, 24 equinos y 6 caninos con la prueba de aglutinación microscópica empleando 12 serovariedades de Leptospira de las más comunes en México; excepto los roedores que se evaluaron contra: siete serovariedades (importantes en este grupo de animales). Las especies valoradas reaccionaron contra una o más serovariedades con el porcentaje de seropositividad siguiente: $50 \%$ de los 
roedores reaccionó contra icterohaemorrhagiae $(37 \%)$, grippotyphosa (14\%), tarassovi (12\%) y canicola (4\%). El $52 \%$ de los bovinos reaccionaron contra hardjoprajitno cepa H89 (45.5\%), hardjoprajitno $(33.1 \%)$, wolffi $(28.6 \%)$ y tarassovi $(9 \%)$. Los equinos presentaron una seropositividad de $70.8 \%$ contra tarassovi (41.6\%), hardjoprajitno (29.1\%), wolffi (12.5\%) y hardjoprajitno cepa H89 (12.5\%). El 100\% de los caninos resultaron seropositivos, a icterohaemorrhagiae cepa palo alto, portland-vere cepa sinaloa y canicola, mientras que contra icterohaemorrhagiae sólo $66.6 \%$.

\section{Diagnóstico}

Comprende el diagnóstico de la bacteria o su genoma. Las concentraciones de Leptospira son bajas en fluidos biológicos por lo que se considera un reto el aislamiento. Además los periodos de leptospiremia suelen ser bastante cortos y durante esta fase los signos clínicos suelen ser muy inespecíficos por lo que la detección es baja. Este tipo de diagnóstico requiere gran experiencia. Los resultados falsos positivos son comunes, principalmente debido a la confusión con hebras de fibrina o restos celulares. No se considera técnica de diagnóstico definitivo, se recomienda no aceptar ni excluir el diagnóstico. Las leptospiras no son teñibles por tinción clásica de Gram, pero se pueden emplear algunas coloraciones que no se utilizan comúnmente en el diagnóstico como la tinción de plata o se puede emplear inmunofluorescencia directa o inmunoperoxidasa (Musso \& Lascola, 2013).

Las leptospiras se cultivan en medios artificiales constituidos por una solución de sales minerales y aminoácidos a los que se añade suero de conejo (medios de Fletcher y Stuart), o albúmina bovina con Tween 80 (medio de Ellinghausen, McCullogh, Harris y Johnson o EMJH). La incubación se realiza a $28-30^{\circ} \mathrm{C}$. Debido a su lento desarrollo, sólo se observa crecimiento después de transcurrida una semana; sin embargo, no debe considerarse el cultivo como negativo sino hasta transcurrido un mes. El crecimiento en medios líquidos se manifiesta por enturbiamiento; y en los medios semisólidos, el desarrollo se inicia a 1-2 cm de la superficie, pero la morfología de la colonia no es característica.

La identificación de la cepa aislada se efectúa mediante técnica de aglutinación microscópica con anticuerpos específicos de serogrupo y serotipo. En los laboratorios de referencia hay anticuerpos monoclonales muy específicos. La identificación se completa con el método molecular de "huellas digitales" (finger-print) y con el análisis de restricción del ADN y uso de endonucleasas (Pumarola \& Rodriguez, 1992; Palaniappan et al., 2002; Ganoza et al., 2006).

La técnica de microagrutinación (MAT) o Test de Martin y Pettit, fue desarrollada aproximadamente hace un siglo en el Instituto Pasteur. Es la técnica de referencia en la que se utilizan suspensiones vivas de Leptospira y hay lectura microscópica en campo oscuro. Su especificidad se refleja en la propiedad del suero del paciente de aglutinar sólo la serovariedad o grupos de serovariedades antígénicamente relacionadas. A pesar que el "MAT" es la prueba serológica de elección, su complejidad limita su aplicación a laboratorios especializados (Terpstra, 1990). Este método presenta algunas desventajas, ya que por su especificidad se requiere de un panel de serovariedades como antígenos diagnósticos que cubran el espectro de las serovariedades que causan la enfermedad en una zona determinada (Chinchilla, Boza \& Sáenz, 1996).

La técnica de ELISA aún no se tiene como una prueba de rutina en los laboratorios de diagnóstico, debido a que la comparación y estandarización en la preparación del antígeno resulta difícil, dado que el antígeno se extrae de diferentes serovariedades, se usan distintos métodos y se analizan por diferentes procedimientos (Chinchilla, Boza \& Sáenz, 1996). Esta técnica ha sido utilizada desde los años noventa. Recientemente se ha incrementado su uso en el diagnóstico en zonas endémicas por su sensibilidad y su capacidad de proveer un diagnóstico temprano. El umbral de detección es de 10-100 leptospiras/ml en sangre u orina. (Piccardeu, 2013). 
Factores de riesgo asociados a la enfermedad en humanos.

Los principales factores de riesgo en Latinoamérica están relacionados con la alta pluviosidad así como con factores sociales como la desigualdad social, ya que poblaciones sin servicios públicos básicos como agua potable, alcantarillado y recolección de basura tienen un riesgo mayor de exposición a leptospirosis. (WHO, 1986; Ospina-Pinto \& Hernández, 2015)

Personas que están expuestas laboralmente a aguas estancadas, labores agrícolas, personal veterinario, ganaderos e inspectores de carne, presentan un alto riesgo de exposición; sin embargo esto depende de las buenas prácticas de bioseguridad que se lleven a cabo durante el contacto con animales portadores o lugares donde pueda encontrarse la bacteria (Kikuti et al, 2012)

\section{Tendencias en el campo de conocimiento.}

Entre 1960 a 2015, de 121 artículos que fueron publicados en el tema; se tiene que 11 artículos corresponden a reportes realizados en Colombia, 8 a Argentina, 11 artículos en Centroamérica, 2 para Chile, 1 a Ecuador, 12 en Perú y 1 a Venezuela; los restantes corresponden a información general acerca de la bacteria y su epidemiología.

Morales, Guzmán \& Beltrán (1978) realizaron el primer estudio de Leptospirosis en Colombia en el que se evaluó el rol epidemiológico de la rata negra (Rattus rattus) en la transmisión de Leptospira serovar pomona en 4 granjas porcícolas infectadas en la región del Valle de Cauca, Colombia. En este trabajo se realizaron 8 aislamientos de 111 muestras de riñón de los cuales 7 corresponden al serovar icterohaemorhagiae y uno a pomona. Aunque la prevalencia es baja los autores resalta la importancia del hallazgo debido a que el serovar icterohaemorhagiae está asociado a la enfermedad de Weil en humanos y a leptospirosis aguda en cerdos.

Lins \& Lópes (1984) examinaron por medio de cultivo y aislamiento un total de 696 animales silvestres en el estado de Pará, Brasil. De 246 animales correspondientes a la especie Proechimys sp., realizaron cuatro aislamientos de Leptospira dos del serovar ballum y dos de un serovar sin identificar. (Lins \& Lopes, 1984), Enrietti en el 2001 realiza un estudio de incidencia en caninos, múridos y suínos en el estado de Paraná, Brasil, en el que se capturaron 50 individuos, 49 de la especie Rattus rattus y uno de la especie Rattus norvegicus. Se utilizaron dos técnicas para el diagnóstico de Leptospira, inoculación en cobayos y cultivo en medio Wenyon. La tasa cruda de positividad fue de $78 \%$. Para la primera técnica se inocularon 45 cobayos de los cuales 25 fueron positivos $(53,19 \%)$ y para el cultivo fueron positivos 31 de los 50 animales capturados (62\%). Al comparar las técnicas los autores pueden establecer que el cultivo es un método más efectivo y menos dispendioso con respecto a la inoculación ya que ésta puede subestimar los resultados por razones como la muerte de los cobayos por diferentes causas (Enrietti, 2001).

Lilenbaum et al. (2002) realizaron un muestreo serológico en el zoológico de Río de Janeiro, Brasil en el que tomaron 77 muestras de 38 especies de animales. Sólo un roedor fue muestreado Sphiggurus villosus y se encontró negativo por la prueba de MAT contra los serovares australis, autumnalis, bratislava, castellonis, ballum, bataviae, canicola, grippotyphosa, hebdomadis, icterohaemorrhagiae, copenhageni, hardjo, pomona, pyrogenes, sejroe, tarassovi y wolffi (Lilenbaum et al., 2002).

Otro estudio realizado en Brasil por Correa et al., publicado en 2004 encontraron que de 302 muestras tomadas en el Parque Zoológico de San Pablo, de las cuales 14 correspondían al orden Rodenthia (7 Rattus norvegicus, 5 Dasyprocta azarae y 2 Hydrochoerus hydrochaeris) Fueron positivos: dos Dasyprocta azarae para el serovar castellonis y tres Rattus norvegicus para el serovar icterohaemorrhagiae (Correa et al., 2004).

Bevilacqua et al. (2004) realizaron una caracterización poblacional y monitoreo de leptospirosis en un hospital veterinario y un fragmento de bosque 
nativo en la provincia de Minas Gerais, Brasil, en donde capturaron 72 animales de la especie Rattus rattus en cercanías del hospital veterinario. En el fragmento de bosque se capturó un total de 32 roedores silvestres de los cuales 16 pertenecían al género Oligoryzomis sp, 11 al género Akodon sp., 3 a Oximicterus sp., uno a Bolomis sp. y uno a Oryzomis sp.. En ninguno de los casos se encontró evidencia de infección por Leptospira, sin embargo, los autores no descartan que estas especies puedan comportarse como reservorios de la enfermedad y atribuyen los hallazgos a un ambiente libre de animales portadores (Bevilacqua et al., 2004).

En el año 2008 Marder et al. realizaron un estudio de prevalencia de leptospira en roedores sinantrópicos y silvestres en la ciudad de Corrientes Argentina en el que se capturaron y muestrearon 100 individuos de la especie Rattus rattus y un individuo de Mus musculus. Por medio de cultivo y microscopía de campo oscuro se estableció que 58 individuos de la especie Rattus rattus al igual que el individuo de Mus musculus fueron positivos. Los autores concluyen que este hallazgo puede representar un gran riesgo para la comunidad debido a la alta prevalencia de la bacteria en los reservorios, sin embargo se desconoce su potencial patógeno ya que no fue reconocida la especie ni los diferentes serovares (Marder et al., 2008).

Silva, Seyffert \& Jouglard (2009) realizaron en Brasil la identificación de infección por Leptospira en Capibaras en una planta de beneficio de Rio Grande do Sul. De las 22 muestras tomadas seis fueron seropositivas con títulos entre 100 y 3200 . Los mayores títulos fueron para el serovar bratislava seguido del serovar australis cepa Hook. También hubo resultados positivos para los serovares panama, patoc, djasiman, copenhageni y autumnalis y se presentó una reacción positiva a Leptonema illini una espiroqueta saprófita genéticamente relacionada con Leptospira (Silva, Seyffert \& Jouglard, 2009).

Conjuntamente Scialfa et al. (2010) en la ciudad de Tandil, Argentina realizan pruebas de microaglutinación (MAT), cultivo y aislamiento de 42 ratas marrones (Rattus norvegicus), encontrando seropositividad en el 52,3\%, (22) para los serovares castellonis, canicola, grippotyphosa, icterohaemorrhagiae y hebdomadis. Los riñones de 25 animales fueron cultivados y se aisló L. interrogans en el 96\% (24) de las muestras. Para los autores esto indica una infección endémica y un potencial riesgo para la salud pública y resaltan la concordancia de los hallazgos con otros realizados previamente en la república Argentina (Scialfa et al., 2010). Grune et al. (2014) aplican la técnica molecular de MLVA (Multiple-Locus Variable number tandem repeat Analysis) para caracterizar 22 cadenas aisladas de roedores urbanos y periurbanos en las provincias de Santa Fe y Entre Ríos, Argentina. Todas las cadenas fueron serotipificadas y arrojaron el mismo resultado que la tipificación por MLVA. 7 cadenas correspondieron a la especie L. interrogans las cuales 5 se tipificaron como serovar copenhageni e icterohaemorrhagiae. Dos de ellas aisladas en ratones periurbanos y tres de la especie Rattus rattus. Dos cadenas aisladas de Rattus norvegicus correspondieron al serovar portlandvere una al serovar canicola y quince a $L$. borgpetersenii serovar castellonis ocho de las cuales fueron aisladas de Mus musculus, una de Rattus sp. y cinco de Rattus rattus. Los autores avalan la técnica de MLVA como una opción para determinar nuevos hospederos silvestres de la bacteria e identificación específica de los serovares para realizar asociaciones epidemiológicas (Grune et al., 2014).

Scialfa, Recavarren, Quintana \& Giamperetti (2015) realizan una comparación entre el PCR y otras técnicas de identificación de leptospira en animales silvestres en la provincia de Buenos Aires, Argentina. De un total de 34 animales fueron capturadas 16 ratas $R$. norvegicus de las cuales resultaron positivas dos por microaglutinación a los serovares grippotyphosa, canicola y casteIlonis; una por cultivo y seis por RT-PCR. En esta investigación se resalta el uso de PCR como una herramienta de alta sensibilidad y útil en el 
diagnóstico de fases tempranas de la enfemedad (Grune, Recavarren, Quintana \& Giamperetti, 2015).

En la actualidad con el objetivo de predecir e interpretar las bases moleculares de la trasmisión de la bacteria, así como encontrar estrategias que permitan realizar un diagnóstico temprano de la enfermedad, la tendencia es utilizar técnicas moleculares para estudiar el comportamiento vectorial de las enfermedades, así mismo para comprender el papel de animales domésticos en el incremento de la frecuencia de la enfermedad.

\section{Conclusiones}

Los estudios en roedores evidencian que aunque estos son portadores de Leptospira, en la mayoría de los casos las especies silvestres tenían una menor prevalencia serológica. En los casos donde se encontraron animales silvestres portadores, con frecuencia los muestreos estaban asociados a explotaciones ganaderas lo que podría indicar que la fuente de infección son los animales domésticos. Si es así, La ampliación de la frontera agrícola es un factor importante para el aumento en la presentación de enfermedades emergentes y reemergentes debido a que se rompen los ciclos normales y se incluyen nuevos reservorios y diseminadores de los patógenos.

Aunque existen diversas técnicas de muestreo, la gran cantidad de serovariedades y sus reacciones cruzadas pueden, en algunos casos, crear confusiones con respecto a la epidemiología y la patogenicidad del microorganismo. En algunas zonas no se cuenta con la batería antigénica necesaria para identificar todas las serovariedades debido a que requieren laboratorios especializados.

Algunos países tras implementar medidas de monitoreo de la enfermedad como el reporte obligatorio generan una mayor cantidad de información. Para enfermedades como la leptospirosis es de vital importancia ya que se puede hacer un seguimiento en el tiempo para implementar medidas de control según su epidemiología.

Es de vital importancia desarrollar estudios en poblaciones silvestres poco intervenidas. Esto con el fin de establecer los posibles ciclos silvestres de infección y la relación hospedero patógeno. A pesar de que en Latinoamérica hay diversas investigaciones en animales silvestres, en Colombia los reportes se reducen a prevalencias en humanos y algunos animales domésticos. Sin análisis más profundos, La relación con la fauna silvestre y los ecosistemas aún es desconocida. En las zonas de ganado bovino hay una mayor prevalencia serológica por lo que deben considerarse estrategias dirigidas a la comprensión del fenómeno.

\section{Agradecimientos}

Los autores agradecen a la Escuela de Ciencias Agrícolas, Pecuarias y del Medio Ambiente de la Universidad Nacional Abierta y a Distancia (UNAD), al departamento de Patología del Instituto Federal del Ceará y al grupo de Estudio de Animales Silvestres de la Universidad Nacional de Colombia, por haber facilitado a cada uno de los autores, espacios de discusión y de búsqueda bibliográfica de la investigación.

\section{Literatura citada}

1. Adler, B. \& Faine, S. (2006) The genus Leptospira. Prokaryotes 7: 294-317.

2. Ashford, D., Kaiser R. \& Spiegel R. (2000) Asymptomatic infection and risk factors for leptospirosis in Nicaragua. Am J Trop Med Hyg. 63: 249-54.

3. Ballard, S.A., Williamson, M., Adler, B., Vinh, T. \& Faine, S. (1986). Interactions of virulent and avirulent leptospires with primary cultures of renal epithelial cells. Journal of Medical Microbiology 21, 59-67.

4. Barbosa, A., Abreu, P., Neves, F., Atzingen, M., Watanabe, M., Vieira, M., Morais, Z., Vasconcellos, S. \& Nascimento, A.( 2006). A newly identified leptospiral adhesin mediates attachment to laminin. Infect. Immun. 74 (11): 6356-64

5. Bevilacqua, P., Carmo, R., Silva, J. \& Giudice, G. M. (2004). Roedores inventariados em hospital veterinário 
e fragmento de mata nativa da Zona da Mata de Minas Gerais, Brasil: caracterização populacional e infecção por Leptospira sp.. Ciência Rural, 34(5), 1519-1523

6. Bharti, A., Nally, J., Ricaldi, J., Matthias, M., Diaz, M., Lovett, M., Levett, P., Gilman, R., Willig, M., Gotuzzo, E .\& Vinetz, J. (2003). Leptospirosis: a zoonotic disease of global importance. The Lancet infectious diseases 3 (12): 757-71.

7. Blood, B., Szyfres, B. \& Moya, V. (1963). Infección por Leptospira Pomona en la cavia de las Pampas (Cavia Pamparum). Boletín de la Oficina Sanitaria Panamericana (OSP); 54 (6):603-09.

8. Bovet, P., Yersin, C., Merien, F., Davis, C. \& Perolat P.(1999). Factors associated with clinical leptospirosis: a population-based case-control study in the Seychelles (Indian Ocean). Int J Epidemiol; 28: 583-90.

9. Bunnell, J., Hice, C., Watts, D., Montrueil, V., Tesh, R. \& Vinetz, J. (2000). Detection of pathogenic Leptospira spp. infections among mammals captured in the Peruvian Amazon Basin region; Am. J. Trop. Med. Hyg. 63 225-228.

10. Cerqueira, G. \& Picardeau, M. (2009). A century of Leptospira strain typing. Infection, Genetics and Evolution. Journal of Molecular Epidemiology and Evolutionary Genetics in Infectious Diseases 9 (5): 760-8.

11. Cespedes, Z.(2005). Leptospirosis: Enfermedad Zoonótica Emergente. Rev. perú. med. exp. salud publica.22, (4): 290-307.

12. Chinchilla, A., Boza, R. \& Saenz, E. (1996). Leptospirosis en humanos. Rev Cost. de Ciencias Médicas.17 (2): $41-60$

13. Cordeiro, F. \& Sulzer, A. (1981). Leptospira interrogans in several wildlife species in southeast Brazil, Pesq. Vet. Bras. 1: 19- 29.

14. Corrêa, S., Vasconcellos, S., Morais, Z., Teixeira, A, Dias, R., Guimarães, M., Ferreira, F. \& Ferreira-Neto J. (2004). Epidemiologia da leptospirose em animais silvestres na Fundação Parque Zoológico de São Paulo. Braz. J. Vet. Res. Anim. Sci. 41:189-193.

15. Corwin, A., Ryan, A., Bloys, W., Thomas, R., Deniega, B. \& Watts, D. (1990). A waterborne outbreak of Leptospirosis ocurred among US militar personnel in Okinawa, Japan. Lnt J Epidemiol; 19(3): 743-8.

16. Dooley, J. \& Ishak, K. Leptospirosis. In: Binford $\mathrm{CH}$, Connor DH. (1976). Pathology of tropical and extraordinary diseases. 1:101-106.

17. Enrietti, M. (2001). Contribuição ao Conhecimento da Incidência de Leptospiras em Murídeos Caninos e suínos no Paraná. Jubilee Volume (1946-2001) : 311 - 342

18. Faine, S., Adler, B., Bolin, C. \& Perolat, P. (1999). Leptospira and leptospirosis. Melbourne, Australia.

19. Ganoza, C., Matthias, M., Collins-Richards, D., Brouwer, K., Cunningham, C., Segura, E., Gilman, R., Gotuzzo, E. \& Vinetz, J. (2006). Determining risk for severe leptospirosis by molecular analysis of environmental surface waters for pathogenic Leptospira. PLoS Med. 3(8): 308

20. Gressler, L., Schafer Da Silva, L., Tonin, A., Azevedo, M., Tales, M. \& González, S. (2009). New serovars of Leptospira interrogans in cavy (Cavia aperea) Brief Communication. Comp Clin Pathol.19:119-120.
21. Grune, S., Passaro, D., Samartino, L., Soncini, A., Romero, G. \& Brihuega, B. (2014). Genotypes of Leptospira spp. strains isolated from dogs in Buenos Aires, Argentina. Rev Argent Microbiol.46(3):201-204.

22. Grune, S., Rago, V., Martínez, M., Uhart, M., FlorinChristensen, M., Romero, G. \&Brihuega, B. (2015). Isolation of a Seawater Tolerant Leptospira spp. from a Southern Right Whale (Eubalaena australis). PLoS Onev.10(12): 1-10.

23. Kikuti, M., Langoni, H., Nobrega, D., Corrêa, A. \& UIImann, L. (2012). Occurrence and risk factors associated with canine leptospirosis. J Venom Anim Toxins incl Trop Dis. 18 (1) : 124-127.

24. Levett, P. (2001). Leptospirosis. Clin. Microbiol. Rev. 14 (2): 296-326

25. Levett, P., Morey, R., Galloway, R. \& Steigerwalt, A. (2006). Leptospira broomii sp. nov., isolated from humans with leptospirosis. Int. J. Syst. Evol. Microbiol. 56 (3): 671-3.

26. Levett, P., Morey, R., Galloway, R., Turner, D., Steigerwalt, A. \& Mayer, L. (2005). Detection of pathogenic leptospires by real-time quantitative PCR. Journal of Medical Microbiology. 54: 45-49.

27. Liceras, J. (1975). Boletín de la Oficina Sanitaria Panamericana (OSP); 410-421

28. Lilenbaum, W., Monteiro, R., Ristow, P., Fraguas, S., Cardoso, V. \& Fedullo, L. (2002). Leptospirosis antibodies in mammals from Rio de Janeiro Zoo, Brazil. Res Vet Sci, 73:319-321.

29. Lins, Z. \& Lopes, M. (1984), Isolation of Leptospira from wild forest animals in Amazonian Brazil. Transactions of the royal society of tropical medicine and hygiene. 78: 124-126.

30. Marder, G., Ruiz, R., Bottinelli, O., Peiretti, H, Zorzo, L., Merino, D., Czernik, G. (2008). Prevalencia de leptospirosis en roedores sinantrópicos de la Cuidad de Corrientes, Argentina. Rev Vet. 19: 150-153.

31. Matthias, M. \& Levett P. (2002). Leptospiral carriage by mice and mongooses on the island of Barbados; West Indian Med. J. 51:10-13.

32. Méndez, C., Benavides, L., Esquivel A., Aldamal, A., Torres, J., Gavaldón, D. \& Meléndez, P. \& Moles, L. (2013). Pesquisa serológica de Leptospira en roedores silvestres, bovinos, equinos y caninos en el noreste de México Rev. Salud Anim. 35(1): 25-32.

33. Montes, D., Rivera, H., Ramírez, M., Ríos, P., Angulo, C. \& Muñoz, K.(2011). frecuencia de infección por leptospira sp. en ardillas nucablanca (sciurus stramineus) en un zoológico de la ciudad de lima. Rev Inv Vet . 22(1): 66-71.

34. Morales, G., Guzmán, V. \& Beltrán, L. (1978). Leptospirosis in Colombia: isolation of Leptospira spp. from the kidneys of brown rats (Rattus norvegicus) trapped on infected piggeries. Trop Anim Health Prod.; 10:121-3.

35. Musso, D. \& Lascola, B. Diagnostic biologique de la leptospirose. (2013). Revue Francophone Des Laboratoires Février. 449: 39-46.

36. Ospina-Pinto, M. \& Hernández, P. (2015). Utilidad de 
las herramientas moleculares para la identificación de Leptospira spp. en muestras humanas, animales y ambientales. Revista cubana de medicina tropical. 67(3) versión electrónica.

37. Palaniappan, R., Chang, Y., Jusuf, S., Artiushin, S., Timoney, J., McDonough, S., Barr, S., Divers, T., Simpson, K., McDonough, P. \& Mohammed, H. (2002). Cloning and molecular characterization of an immunogenic LigA protein of Leptospira interrogans. Infect. Immun. 70 (11): 5924-30.

38. Percy, D. \& Barthold, S. (2007), Pathology of laboratory rodents and rabbits. 3rd edition. Ames (IA): Blackwell Publishing Professional. p. 125-78.

39. Pereira, M. \& Andrade, J. (1990). Human Leptospirosis in a slum area in the City of Rio de Janeiro, Brazil. Mem Inst Oswaldo Cruz; 85:47-52.

40. Petrakovsky, J., Bianchi, A., Fisun, H.,Nájera-Aguilar, P. \& Pereira, M. (2014). Animal Leptospirosis in Latin America and the Caribbean Countries: Reported Outbreaks and Literature Review (2002-2014). Int. J. Environ. Res. 11(10): 10770-10789.

41. Picardeau, M., Brenot, A. \& Saint Girons, I. ( 2001). First evidence for gene replacement in Leptospira spp. Inactivation of $\mathrm{L}$. biflexa flaB results in non-motile mutants deficient in endoflagella. Molecular Microbiology. 40:189-199.

42. Piccardeu, M. (2013). Diagnosis and epidemiology of leptospirosis. Médecine et maladies infectieuses. 43: 1-9

43. Pumarola, A. \& Rodríguez, A. (1992). Leptospira En: Perea EJ (ed). Enfermedades infecciosas y microbiología clínica. 2a ed. Barcelona: Doyma; 729-734.

44. Riedemann, S. \& Zamora, X. (1982). Leptospirosis en pequeños roedores en el área rural de Valdivia, Zbl. Vet. Med. B. 29: 764- 768.

45. Sacsaquispe, C., Glenny, A. \& Cespedes, Z. (2003). Estudio preliminar de leptospirosis en roedores y canes en salitral, Piura-1999. Rev. perú. med. exp. 20(1): 39-40.
46. Sandow, K. \& Ramírez, W. (2005). Leptospirosis REDVET. Revista Electrónica de Veterinaria, VI Junio.

47. Scialfa, E., Bolpe, J., Bardon, J., Ridao, G., Gentile, J. \& Gallicchio, O. (2010). Isolation of Leptospira interrogans from suburban rats in Tandil, Buenos Aires. Rev Argent Microbiol. 42: 126-128.

48. Scialfa, E., Recavarren, M., Quintana, S. \& Giamperetti, S. (2015). Comparison between real-time PCR, serology and culture in leptospirosis from samples of wild animals trapped in Buenos Aires Province Argentina. Rev Argent de Zoonosis y Enfermedades Infecciosas Emergentes. 10(2): 24-28.

49. Silva, E., Seyffert, N. \& Jouglard, S. (2009). Soroprevalenciada de infeccao leptospiral em capivaras (Hydrochoerus hydrochaeris) abatidas em um frigorífico do Rio Grande do Sul. Pesqui Vet Bras 29:174-176

50. Terpstra, W. (1990). Enzymatic detection systems for Leptospiral antibodies, antigen and nucleic acids. WHO/FAO Collaborating Center for reference and Research on Leptospirosis. Holland.

51. Vanasco, N., Sequeira, M., Sequeira, G. \& Tarabla, H. (2003). Associations between leptospiral infection and seropositivity in rodents and environmental characteristics in Argentina. Prev Vet Med. 60(3):227-235

52. WHO. (1986) Report of the WHO consultation the development of National Programmes for the prevention and control of Leptospirosis. Sapporo Japan 1984.

53. Zamora, J. (1998). Riesgo epidemiológico de la fauna silvestre en problemas de salud. X Congreso de Medicina Veterinaria, Simposios: 127- 151.

54. Zamora, J. Riedemann, S. (1999). Aislamiento y sobrevivencia de Leptospiras en tejido renal de roedores silvestres, Arch. Med. Vet. 31: 103-107.

55. Zamora, J., Riedemann, S. \& Cabezas, X. (1995). Leptospirosis de los roedores silvestres en el área rural de valdivia. pesquisa de $\mathrm{L}$. interrogans mediante inmunofluorescencia e inmunoperoxidasa, Arch. Med. Vet. 27: 115- 118.

\section{Conflicto de Intereses}

Los autores declaran no tener ningún conflicto de intereses 